\title{
Graphene oxide as a nanocarrier for controlled release and targeted delivery of an anticancer active agent, chlorogenic acid
}

\begin{abstract}
We have synthesized graphene oxide using improved Hummer's method in order to explore the potential use of the resulting graphene oxide as a nanocarrier for an active anticancer agent, chlorogenic acid (CA). The synthesized graphene oxide and chlorogenic acid-graphene oxide nanocomposite (CAGO) were characterized using Fourier transform infrared (FTIR) spectroscopy, thermogravimetry and differential thermogravimetry analysis, Raman spectroscopy, powder X-ray diffraction (PXRD), UV-vis spectroscopy and high resolution transmission electron microscopy (HRTEM) techniques. The successful conjugation of chlorogenic acid onto graphene oxide through hydrogen bonding and $\pi-\pi$ interaction was confirmed by Raman spectroscopy, FTIR analysis and X-ray diffraction patterns. The loading of CA in the nanohybrid was estimated to be around $13.1 \%$ by UV-vis spectroscopy. The release profiles showed favourable, sustained and $\mathrm{pH}$-dependent release of CA from CAGO nanocomposite and conformed well to the pseudo-second order kinetic model. Furthermore, the designed anticancer nanohybrid was thermally more stable than its counterpart. The in vitro cytotoxicity results revealed insignificant toxicity effect towards normal cell line, with a viability of $>80 \%$ even at higher concentration of $50 \mu \mathrm{g} / \mathrm{mL}$. Contrarily, CAGO nanocomposite revealed enhanced toxic effect towards evaluated cancer cell lines (HepG2 human liver hepatocellular carcinoma cell line, A549 human lung adenocarcinoma epithelial cell line, and HeLa human cervical cancer cell line) compared to its free form.
\end{abstract}

Keyword: Graphene oxide; Drug delivery; Sustained release; Nanocomposite; Anticancer property 